\title{
ON CREDIBILITY OF SIMULATION STUDIES OF TELECOMMUNICATION NETWORKS
}

\author{
K. Pawlikowski*, H.-D. Joshua Jeong and J.-S. Ruth Lee \\ Department of Computer Science \\ University of Canterbury \\ Christchurch, New Zealand \\ Email: k.pawlikowski@cosc.canterbury.ac.nz
}

January $28,2001^{\dagger}$

\section{KEYWORDS}

Stochastic Simulation, Simulation of Telecommunication Networks, Credibility of Simulation.

\section{ABSTRACT}

In telecommunication networks, as in many other areas of science and engineering, proliferation of computers as research tools has resulted in the adoption of computer simulation as the most commonly used paradigm of scientific investigations. This, together with a plethora of existing simulation languages and packages, has created a popular opinion that simulation is mainly an exercise in computer programming. In new computing environments, programming can be minimised, or even fully replaced, by the manipulation of icons (representing pre-built programming objects containing basic functional blocks of simulated systems) on a computer monitor. One can say that we have witnessed another success of modern science and technology: the emergence of wonderful and powerful tools for exploring and predicting the behaviour of such complex, stochastic dynamic systems as telecommunication networks.

But this enthusiasm is not shared by all researchers in this area. An opinion is spreading that one cannot rely on the majority of the published results on performance evaluation studies of telecommunication networks based on stochastic simulation, since they lack credibility. Indeed, the spread of this phenomenon is so wide that one can speak about a deep crisis of credibility. In this paper, this claim is supported by the results of a survey of over 2200 publications on telecommunication networks in recent proceedings of the IEEE INFOCOM and such journals as the IEEE Transactions on Communications, the IEEE/ACM Transactions on Networking, and the Performance Evaluation Journal.

\footnotetext{
*This work was partially supported by the University of Canterbury's Grant no. U6301

${ }^{\dagger}$ Status: a revision of the paper submitted to the IEEE Communications Magazine in April 2000
} 
Our discussion focuses on two important necessary conditions of a credible simulation study: use of appropriate pseudo-random generators of independent uniformly distributed numbers, and appropriate analysis of simulation output data. Having considered their perils and pitfalls, we formulate guidelines that, if observed, could help to assure a basic level of credibility of simulation studies of telecommunication networks.

\section{Introduction}

The last decade of the twentieth century will be remembered as a time when computers found their place in primary schools and in private homes, and became ordinary items of equipment on desks in offices and businesses. This is also a time when the computing paradigm has begun its drift from computer networks to network computing. There is enormous interest, both in industry and academia, in creating an AAA network, a world-wide computer network able to offer Any information service, accessible from Any place and at Any time. Before it happens, scientists and engineers will have to investigate many challenging problems of network technology, and evaluate possible solutions. These research activities are certainly accelerated by achievements in the area of scientific computing, with various easy-to-use software packages specifically designed for conducting performance evaluation studies of telecommunication networks.

In the area of telecommunication networks, as in many other areas of science and engineering, proliferation of computers as research tools has resulted in the wide adoption of computer simulation as a new paradigm of scientific investigation, in addition to the two traditional ones: theoretical studies and experimentation. Various user-friendly simulation packages offer sophisticated graphical user interfaces, animation of simulated processes, etc. This has led to a belief that simulation is mainly an exercise in computer programming. Further, this programming can be greatly simplified, since there is a plethora of simulation languages which reduce designing simulation models of telecommunication networks to placing icons (representing basic functional blocks of networks) in appropriate locations on a computer monitor, and then initiating simulation by selecting an appropriate button from a menu bar.

One can say that we have witnessed another success of modern science and technology: the emergence of wonderful and powerful tools for exploring and predicting the behaviour of such complex, stochastic dynamic systems as telecommunication networks. In fact, stochastic discreteevent simulation has already become a commonly used tool of scientists and engineers in this area, contributing to over $51 \%$ of all published research results; see Figure 1. The figure depicts data obtained from a survey of all papers published in proceedings of the IEEE INFOCOM between 1992 and 1998 (1192 papers; ranging between 156 and 177 papers per year), the IEEE Transactions on Communications (1996-1998; 657 papers), the IEEE/ACM Transactions on Networking (19961998; 223 papers), and the Performance Evaluation Journal (1996-1998; 174 papers). A total of 2246 papers were surveyed.

This enthusiasm is not shared by all simulation developers and users. Some claim that stochastic simulation as a performance evaluation tool of various dynamic systems, including telecommunication networks, is misused, and that the spread of this phenomenon is so wide that one can speak about a deep credibility crisis. It is even claimed that one cannot rely on the majority of the published results of performance evaluation studies of dynamic systems based on stochastic simulation. Editorials such as [5] or panel discussions of specialists on the topic, such as those organized at the Winter Simulation Conference in 1994 and 1996, or at the IEEE INFOCOM in 1996, have not changed the situation. 




Figure 1: Proportion of papers that reported results based on stochastic simulation; obtained on the basis of the survey of 2246 papers published in the Proc. of the IEEE INFOCOM (19921998), the IEEE Transactions on Communications (1996-1998), the IEEE/ACM Transactions on Networking (1996-1998) and the Performance Evaluation Journal (1996-1998).

In this paper we investigate the motivation and the validity of such claims. Having narrowed our interest to the application of stochastic discrete-event simulation in performance evaluation studies of telecommunication networks, we look more closely at two important necessary conditions of a credible simulation study: use of appropriate pseudo-random generators of independent uniformly distributed numbers, and appropriate analysis of simulation output data. Having considered their perils and pitfalls, we formulate guidelines that, if observed, could help to assure a basic level of credibility of simulation studies of telecommunication networks.

\section{The Issue of Credibility}

P. J. Kiviat in his opening address of the Summer Computer Simulation Conference SCSC'90 [9] stated that "... succeeding in simulation requires more than the ability to build useful models ...". Some experts assess that the modelling phase of simulation consumes only 30-40\% of the total effort in most successful simulation projects [13]. The first necessary step of any performance evaluation studies based on stochastic simulation is to use a valid simulation model. In the case of telecommunication networks, it means a valid conceptual model of the network, based on appropriate assumptions about the network's internal mechanisms, limitations, stochastic characteristics of processes which will be simulated, etc. A good discussion of general guidelines on how to build valid simulation models can be found, for example, in [12]. However, this is only the first step for ensuring the credibility of the final results of simulation studies.

The next step is to ensure that the valid simulation model is used in a valid simulation experiment. At this stage, two main issues that have to be addressed for ensuring validity of any stochastic simulation-based experiment are: (i) application of appropriate elementary source(s) of randomness, and (ii) appropriate analysis of simulation output data. Let us look closer at these two issues. 


\section{Elementary Sources of Randomness}

It is a generally accepted and commonly used practise today to use algorithmic generators of (pseudo-random) uniformly distributed numbers as elementary sources of randomness in stochastic simulation. The theoretical foundations of PRNGs are well established (see, for example, [11] and over the last 50 years many different PRNGs, that have passed rigorous theoretical tests, have been proposed. Practically all of them can be classified as linear congruential PRNGs (LC-PRNGs), and generate periodic sequences of numbers. The most popular generators of simulation practise have belonged to a class of multiplicative LC-PRNGs, based on recursive algorithms in integer modulo $\mathrm{M}$ arithmetic [2].

In today's world of 32-bit computers, multiplicative LC-PRNGs with the modulus $M=2^{31}-1$ have focused special attention and, following exhaustive analysis, about 20 of them have been recommended as acceptable sources of independent and uniformly distributed pseudo-random numbers. These are the generators that have been used, for example, in GPSS (version H and PC), SIMSCRIPT II.5, SIMAN and SLAM II [12]. Thus, one would expect there to be no problem with selecting a good PRNG.

Unfortunately, this is only partially true. Conscientious users of PRNGs should be aware of potentially very serious problems with using PRNGs in real-life applications. One is that recent achievements of electronic technology have made PRNGs with cycles in the order of $2^{32}$ obsolete in all but very short lasting simulation studies. Today, a standard workstation equipped with a CPU operating with a speed of a few hundred MHZ can generate all numbers of a $\bmod \left(2^{31}-1\right)$ PRNG in a dozen minutes. And ... this is not the final evidence of Moore's law in action: $1 \mathrm{GHz} \mathrm{PCs}$ have been already announced ([19]). Thus, when planning a simulation lasting longer than a few minutes of CPU time, one obviously needs PRNGs of much longer cycles than those that would have been satisfactory only a couple of years ago.

Note that simulation studies of multimedia networks (fed by streams of teletraffic modelled by strongly autocorrelated, or even selfsimilar long-range dependent, processes) can require very long simulations to obtain the final results with an acceptably small statistical error or, in other words, to collect representative samples of simulation output data (see the next section).

The use of PRNGs with adequately long cycles is also strongly advocated by recently established theoretical restrictions on the number of pseudo-random numbers from one PRNG that can be used in a single simulation. For example, if one is concerned with two dimensional uniformity of pseudo-random numbers generated by a PRNG with cycle length $\mathrm{L}$, then one should not use more than $8 \sqrt[3]{L}$ numbers from this PRNG during one simulation [15].

Fortunately, recent advances in the area of PRNGs have given us generators that, in a foreseeable future, should be adequate for simulations demanding even very long CPU time. A number of Multiple Recursive LC-PRNGs, and Combined Multiple Recursive LC-PRNGs, of cycles between $2^{185}$ to $2^{377}$, can be found in [14], together with their portable implementations. Their virtual randomness has been found to be satisfactory in up to 32 dimensions.

Theoretical advances in another class of PRNGs, based on recursions in polynomial arithmetic and known as Generalized Feedback Shift Register (GFSR) PRNGs, have led to even more amazing findings. A twisted GFSR-PRNG, known as the Mersenne Twister, with a super astronomical cycle of $2^{19937}-1$, and good virtual randomness in up to 623 dimensions (!), for up to 32-bit accuracy, has been proposed in [20]. Its portable implementation in C, for 32-bit machines, appears to be faster than a standard PRNG used in the ANSI C rand() function ${ }^{1}$; see

\footnotetext{
${ }^{1}$ The ANSI $\mathrm{C}$ rand() function uses LC-PRNG in integer arithmetic with $M=2^{31}, 1103515245$ as the multiplier, and 12345 as the additive constant
} 
www.math.keio.ac.jp/matumoto/emt.html for the latest news regarding the Mersenne Twister.

Thus, at this stage, there exist PRNGs of acceptable quality, able to be used as sources of elementary randomness in stochastic simulations. However, this does not mean that all problems related with PRNGs have been solved. For example, one should be careful with using uniformly distributed pseudo-random numbers from the same generator in distributed and/or parallel simulation, because of potential correlations existing between disjoint sub-streams of consecutive numbers [7]. In such types of simulation one should use PRNGs with extreme caution, since the final results can be very misleading if correlations hidden in the random numbers and in the simulated system interfere with each other..

In the case of traditional, non-distributed and non-parallel simulation on single processors, one has to be careful too. Uncontrolled distribution of various computer programs has resulted in the uncontrolled proliferation of really poor PRNGs, of clearly unsatisfactory or unknown quality. Thus, the advice given by D. E. Knuth in 1969 is even more important today, in the era of the Internet: “... replace the random generators by good ones. Try to avoid being shocked at what you find ...”, [11]. A longer list of useful practical guidelines on how to use, or not use, PRNGs in simulation studies can be found, for example, in [8], together with the advice that "... it is better to use an established generator that has been tested thoroughly than to invent a new one”.

\section{Simulation Output Data Analysis}

Any stochastic computer simulation, in which random processes are simulated, has to be regarded as a (simulated) statistical experiment and, because of that, application of statistical methods of analysis of (random) simulation output data is mandatory. Otherwise, J. Kleijnen of the University of Tilburg, the Netherlands, warns that “... computer runs yield a mass of data but this mass may turn into a mess <if the random nature of such output data is ignored, and then $>$... instead of an expensive simulation model, a toss of the coin had better be used", [10]. Von Neumann, having noticed a similarity between computer simulators producing random output data and a roulette, coined the term Monte Carlo simulation.

Statistical error associated with the final result of any statistical experiment or, in other words, the degree of confidence in the accuracy of a given final (point) estimate, is commonly measured by the corresponding confidence interval (CI) at a given confidence level (CL), i.e. by the interval CI expected to contain an unknown value with the probability CL. In any correctly implemented simulation, the width of a CI will tend to shrink with the number of collected simulation output data, i.e. with the duration of the simulation.

Two different scenarios exist for determining the duration of a given stochastic simulation. Traditionally, the length of the simulation experiment was set as an input to the simulation programs. In such a fixed-sample-size scenario, where the duration of the simulation is pre-determined either by the length of the total simulation time or by the number of collected output data, the magnitude of the final statistical error of the results is a matter of luck. This is no longer an acceptable approach !

Modern methodology of stochastic simulation offers an attractive alternative solution, known as the sequential scenario of simulation or, simply, sequential simulation. Today, the sequential scenario is recognised as the only practical approach allowing control of the error of the final results of a stochastic simulation, since "... no procedure in which the run length is fixed before the simulation begins can be relied upon to produce a confidence interval that covers $<$ the true value $>$ with the desired <confidence level>" [12]. Sequential simulation follows a sequence of consecutive checkpoints at which the accuracy of estimates, conveniently measured by the relative 
statistical error (defined as the ratio of the half-width of a given CI and the point estimate), is assessed. The simulation is stopped at a checkpoint at which the relative error of estimates falls below an acceptable threshold.

There is no problem with running a simulation sequentially if one is interested in the performance of a simulated network within a well-specified period of (simulated) time and the simulation output data obey the central limit theorem ${ }^{2}$; for example when studying throughput in a network during the 24 hours of its operation. This is the so-called terminating or a finite time horizon simulation. In our example, one would simply need to repeat the simulation (of the 24 hours of the network's operations) an appropriate number of times, using different, statistically independent sequences of pseudo-random numbers as sources of elementary randomness in different replications of the simulation. This ensures that the sample of collected output data (one data item per replication) can be regarded as representing independent and identically distributed random variables, and confidence intervals can be calculated using standard, well-known methods of statistics.

When one is interested in studying the behaviour of networks in steady-state, then the scenario is more complicated. First, since a steady-state is theoretically reachable by a network after an infinitely long period of time, the problem lies in the execution of a steady-state simulation within a finite period of time. Various methods of approaching that problem, in the case of analysis of mean values, are discussed, for example, in [22]. Each of them involves some approximations. Most of them (except the so-called method of regenerative cycles) require that data collected at the beginning of a simulation, during initial warm-up periods, are not used to calculate steadystate estimates. If they are included in further analysis, they can cause a significant bias of the final results; see, for example, [28]. Determination of the lengths of warm-up periods can require quite elaborate statistical techniques $[6,22]$. When this is done, one is left with a time series of (heavily) correlated data, and with the problem of estimating the confidence intervals from such data. However, although the search for robust techniques of output data analysis for steady state simulation continues [24], reasonably satisfactory implementations of basic procedures for calculating steady-state confidence intervals of, for example, mean values and quantiles have already been published; for example, see [22] and [26], respectively.

There are claims that sequential steady-state simulation, and the associated problem of analysis of statistical errors, can be avoided by running simulation experiments sufficiently long to ensure that any influence of the initial states of simulation becomes negligible. While such a brute force approach to stochastic steady-state simulation can sometimes lead to acceptable results ${ }^{3}$, one should be cautious of the statistical accuracy of the final results even in such cases.

Firstly, such very long simulation studies could be relied upon only if PRNGs of appropriate (very long) cycles were used to avoid repeating the sequence of generated pseudo-random numbers, since it can introduce superficial correlations between simulated processes. It should also be remembered that in stochastic discrete-event simulation, collecting a sufficiently large sample of data is more important than simply running the simulation over a long period. The CPU time spent on the simulation of telecommunication processes during which no event of interest is recorded does not have a direct influence on the statistical accuracy of the estimates dependent on the occurrence of these events. What matters is the number of events recorded. For example, when analysing rare events, a minimum number of the rare events must be recorded during a given sim-

\footnotetext{
${ }^{2}$ The central limit theorem says that the average of a large number of output data has, in the limit (as the sample size tends to infinity), a normal probability distribution. This theorem is not applicable to, for example, output data governed by some heavy-tailed distributions.

${ }^{3}$ Some researchers execute their network simulations for a week, or longer, to get the results, they claim, representing steady-state behaviour of simulated networks.
} 
ulation to ensure that the sample of output data is representative. This phenomenon is illustrated in Table 1, which shows that estimates of the mean delays of packets (obtained from a simulation of a DQDB network with 20 stations, over 1500000 time slots) can still be associated with as high a relative statistical error as $43 \%$ or more [16]. The explanation, given in [16], is clear: during this simulation many simulated time slots were idle. When there was no packet for transmission, no packet delay was measured, and no output data item was collected. Instead of stopping the simulation after a fixed time (here: after 1500000 time slots), it should be done when the statistical errors of all estimated mean packet delays become acceptably low.

\begin{tabular}{|c|c|c|c|}
\hline \multirow{2}{*}{ Station } & \multicolumn{3}{|c|}{ Traffic load } \\
\cline { 2 - 4 } & $20 \%$ & $60 \%$ & $90 \%$ \\
\hline 1 & 0.090 & 0.048 & 0.101 \\
\hline 2 & 0.059 & 0.047 & 0.090 \\
\hline 3 & 0.103 & 0.055 & 0.120 \\
\hline 4 & 0.131 & 0.057 & 0.101 \\
\hline 5 & 0.110 & 0.038 & 0.137 \\
\hline 6 & 0.131 & 0.049 & 0.128 \\
\hline 7 & 0.109 & 0.080 & 0.084 \\
\hline 8 & 0.069 & 0.056 & 0.119 \\
\hline 9 & 0.081 & 0.080 & 0.108 \\
\hline 10 & 0.135 & 0.076 & 0.157 \\
\hline 11 & 0.208 & 0.062 & 0.172 \\
\hline 12 & 0.186 & 0.091 & 0.212 \\
\hline 13 & 0.226 & 0.091 & 0.145 \\
\hline 14 & 0.314 & 0.143 & 0.106 \\
\hline 15 & $*$ & 0.102 & 0.202 \\
\hline 16 & $*$ & 0.145 & 0.203 \\
\hline 17 & $*$ & 0.211 & 0.243 \\
\hline 18 & $*$ & 0.248 & 0.430 \\
\hline 19 & $*$ & $*$ & $*$ \\
\hline
\end{tabular}

Table 1: Relative error of mean packets delays at stations of a DQDB network with 19 transmitting stations. All results obtained at 0.95 confidence level. The simulation lasted for 1500000 (simulated) time slots. Cases where the relative errors were not assessed, because too few observations were collected, are marked by * (from [16]).

Stopping stochastic simulation too early can give misleading, or at least inconclusive, results, as illustrated in Figure 2. The figure shows the final results obtained from a sequential steady-state simulation of the same model, but with output data analysis stopped when all estimates reached a relative statistical error not exceeding 25\% (Figure 2a) or 1\% (Figure 2b). In this context, one should certainly question the sense of drawing conclusions on the basis of results with high statistical errors, or results for which statistical errors were not measured at all.

Obtaining final simulation results with small statistical errors is also important in comparative performance evaluation studies of alternative solutions. This phenomenon is illustrated by Figure 3 , which shows the results of the comparative analysis of three alternative versions of a reservation protocol for a wireless ATM network with integrated services; [27]. The results were obtained by 


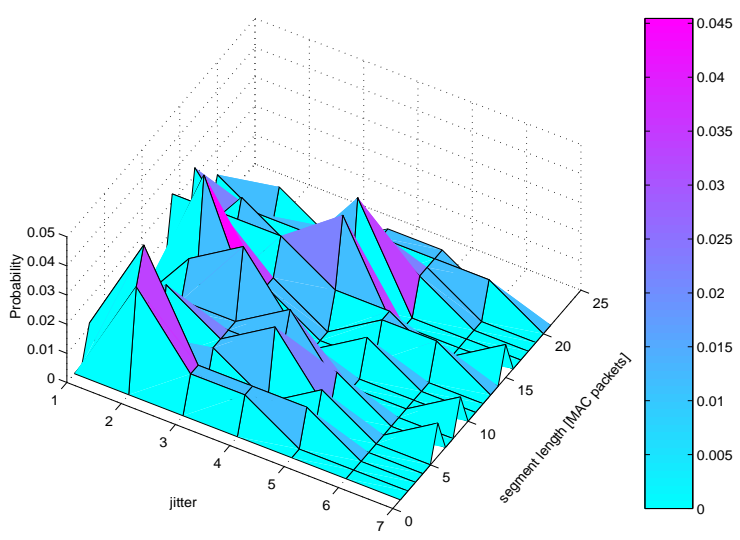

(a) Results with relative statistical errors of $25 \%$ or less

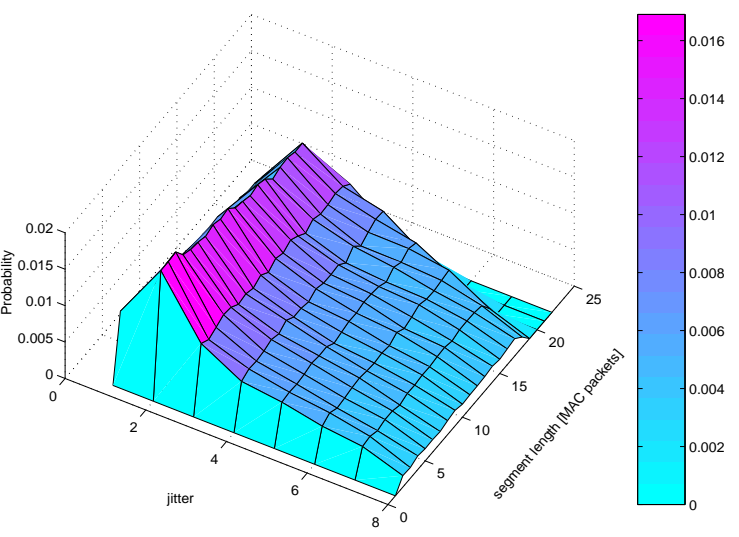

(b) Results with relative statistical errors of $1 \%$ or less

Figure 2: Example showing influence of statistical errors on the quality of simulation results. The assumed confidence level=0.9. Evaluation of a Medium Access Control protocol of a mobile communication network (from [4]).

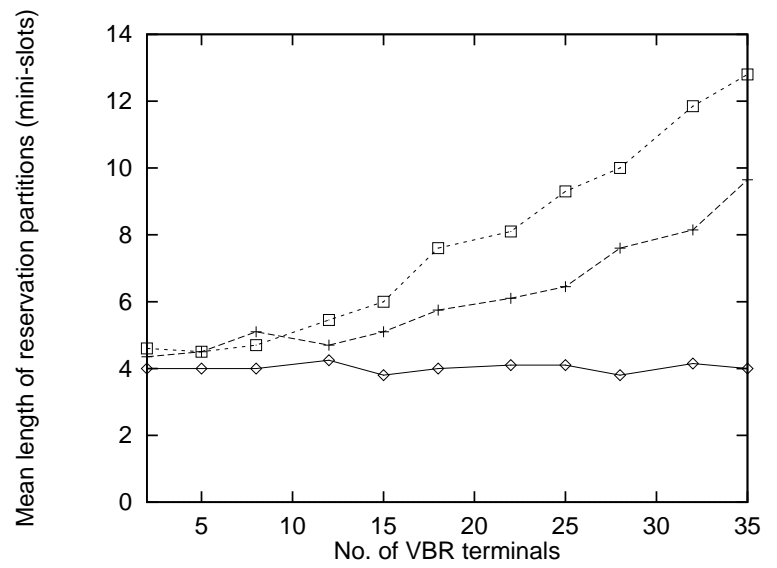

(a) Results with relative statistical errors of $10 \%$ or less

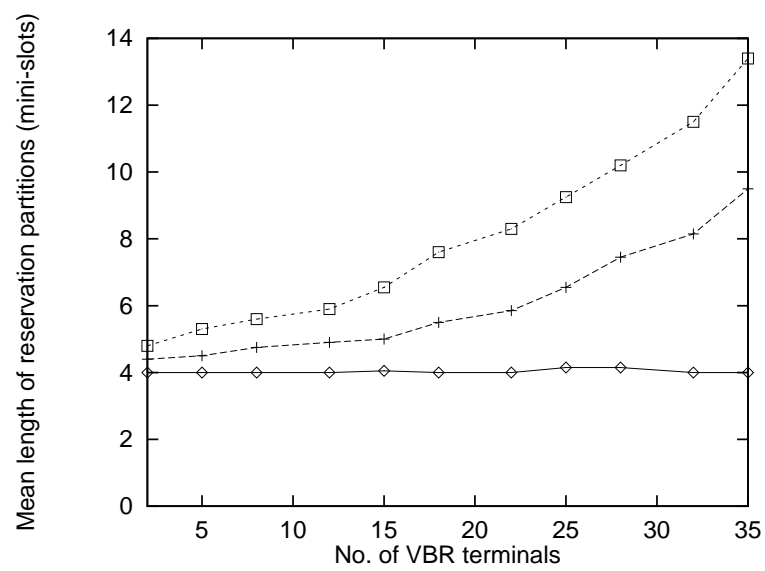

(b) Results with relative statistical errors of $5 \%$ or less

Figure 3: Example showing influence of statistical errors on the final simulation results. Evaluation of three alternative versions of a reservation protocol for a wireless ATM network with integrated services; the assumed confidence level $=0.95$ (from [27])

means of sequential simulation, continued until the relative error of the estimates became as small as $10 \%$ (in Figure 3a), or as small as $5 \%$ (in Figure 3b). One can see that results obtained with too large a statistical error can lead to false conclusions. In this particular case, using the results with an error of $10 \%$, one could erroneously conclude that two of the three versions of the investigated protocol are equivalent as long as no more than 10-12 VBR terminals are used.

Unfortunately, sequential stochastic simulation is not very popular among designers of commercial simulation packages, with an overwhelming majority of them promoting analysis of output 
data only after the simulation is finished. Such packages as, e.g., Arena (by Rockwell Software; see www.arenasimulation.com), Prophesy (by Abstraction Software; see www.abstraction.com), QNAP2 (by Simulog; see www.simulog.fr) or SIMSCRIPT II.5 (by CACI; see www.caciasl.com) are among the few exceptions. To this list of (commercial) packages able to execute stochastic simulation sequentially, one could also add a few packages designed at universities and offered as freeware for non-profit research organisations. One such package is Akaroa-2 [3], designed at the University of Canterbury, in Christchurch, New Zealand.

\section{Crisis}

It would probably be difficult to find a computer scientist or telecommunication engineer today who has not been trained in how to assess and minimise errors inevitably associated with statistical inference. At the same time, the results of our survey of recent publications showed that a surprisingly large proportion of papers reporting simulation-based results did not care about the random nature of output data generated by stochastic simulation. Such "don't care" papers (represented in Figure 4(a)-4(d), by white bars) constituted 76.6\%, 79.05\%, 71.6\% and 68.6\% of papers reporting simulation-based results and published in the Proceedings of the IEEE INFOCOM, the IEEE Transactions on Communications, the IEEE/ACM Transactions on Networking and the Performance Evaluation Journal, respectively. When the type of simulation was reported, then terminating simulation (TS) and steady-state simulation (SS) appeared to be almost equally often applied in the papers published in conference proceedings, while TS dominated in journal publications. Nevertheless, the vast majority of papers did not contain any information about the time-horizon over which the performance of simulated systems was analysed; c.f. NN bars in Figure 4(a)- 4(d).

As Figure 5 shows, on average, about $76.45 \%$ of authors of simulation-based papers on telecommunication networks were not concerned with the random nature of the results obtained from their stochastic simulation studies and either did not care to mention that their final results were outcomes of appropriate statistical analyses or ... reported purely random results. Let us add that Figures 4 and 5 were obtained assuming that papers simply reporting averaged results (say, averaged over a number of replications), but without any notion of statistical errors, were included into the category of papers "with statistically analysed results".

While one can claim that the majority of researchers investigating performance of networks by stochastic simulation simply may not mention that their final results have been subjected to an appropriate statistical analysis, this is not an acceptable practise. Probably everybody agrees that performance evaluation studies of telecommunication networks should be regarded as a scientific activity in which one tests hypotheses on how these complex systems would work if implemented, including even their possibly most critical conditions. However, if this is a scientific activity, then one should follow the scientific method, a generally accepted methodological principle of modern science ([21], Chapter IX). This method says that any scientific activity should be based on controlled and independently repeatable experiments; see, for example, [25], Section 15.

Through many repetitions of a non-sequential simulation one can eventually obtain the final results with acceptably small statistical errors. Thus, using non-sequential simulation, it is still possible to control the error of final results. However, the real problem is that the vast majority of simulation experiments reported in telecommunication network literature are not repeatable. A typical paper contains very little or no information about how a simulation was run. Our survey revealed that authors of almost $52 \%$ of the papers reporting simulation-based results did not even 


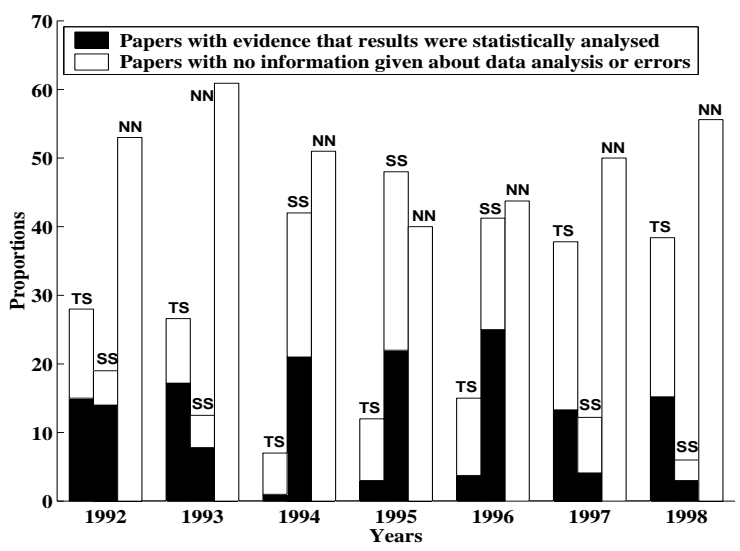

(a) Proceedings of the INFOCOM

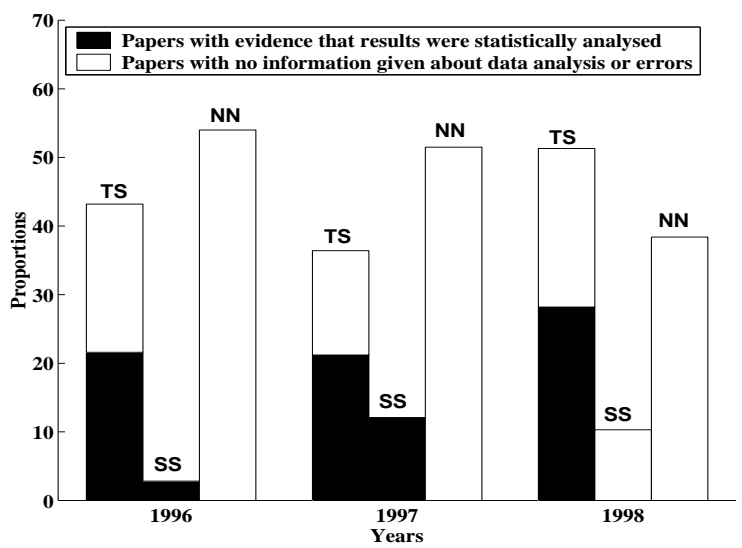

(c) IEEE/ACM Transactions on Networking

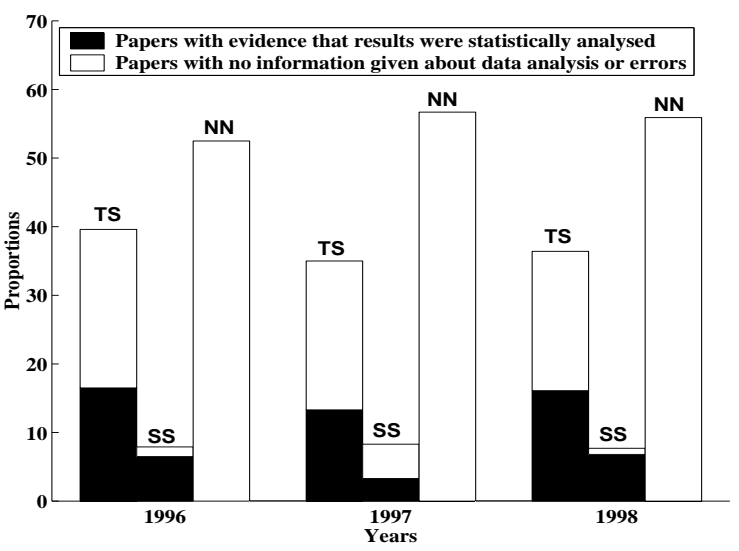

(b) IEEE Transactions on Communications

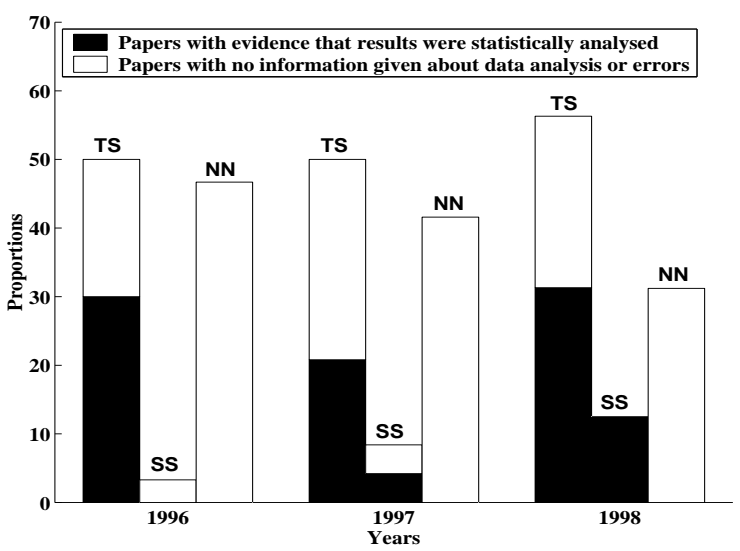

(d) Performance Evaluation Journal

Figure 4: Histograms of papers reporting simulation-based results, as published in (a) the Proceedings of the INFOCOM, (b) the IEEE Transactions on Communications, (c) the IEEE/ACM Transactions on Networking, and (d) the Performance Evaluation Journal. TS: papers reporting results of terminating simulation; SS: papers reporting results of steady-state simulation; NN: papers with no information about the type of simulation executed and with no information about output data analysis or errors

inform the reader whether their results came from terminating or steady-state simulation.

While the principles of the scientific method are generally observed by researchers conducting experiments in such natural sciences as biology, medicine or physics, the crisis of credibility of scientific outcomes is not limited to the area of telecommunication networks but has spanned over the whole area of computer science, as well as electronic and computer engineering; despite such early warnings as that in 1990 by B. Gaither, then Editor-in-Chief of the ACM Performance Evaluation Review, who, being concerned about the way in which stochastic simulation was used then, wrote that he was unaware of "... any other field of engineering or science <other than computer science and engineering $>$ where similar liberties are taken with empirical data ...” [5]. What can be done to change the attitude of writers (who, of course, are also reviewers) of papers 




Figure 5: Papers with statistically analysed output data as a proportion of papers reporting simulation-based results

reporting simulation studies of telecommunication networks? The consequences of drawing potentially not fully correct, or false, conclusions about a network performance can be huge. On the other hand, thorough prediction of networks' performance could make such disasters as the 1990 failure of AT\&T's entire long distance network avoidable. An interesting discussion of the danger associated with modern computer and network technology can be found, for example, in [17].

\section{A Solution?}

The credibility crisis of simulation studies of telecommunication networks could be resolved if some obvious guidelines for reporting the results of simulation studies were adopted.

Firstly, the reported simulation experiments should be repeatable. This should mean that information about

- the PRNG(s) used during the simulation and

- the type of simulation

is provided. The former should be either explicitly specified in a given paper or in the documentation of the simulation package used in the study. In the latter, in the case of a terminating simulation, its time horizon should be specified.

Secondly, one should specify:

- the method of analysis of simulation output data, and

- the final statistical errors associated with the results.

A satisfactory level of credibility of the final simulation results cannot be obtained without assessing their statistical errors, although sometimes, in preliminary studies, it can be acceptable to reduce the randomness of the output results of a simulation simply by repeating the simulation a number of times and averaging the results over replications. D. Knuth wrote that "... the most prudent policy for a person to follow is to run each Monte Carlo program < or stochastic simulation of a telecommunication network $>$ at least twice, using quite different sources of pseudo-random numbers, before taking the answers of the program seriously"; [11]. 
As mentioned, to achieve full credibility of a simulation one needs to develop valid simulation models and use them in valid simulation experiments. The former includes accurate procedural representation of the simulated system's functionality as well as semantic and syntactical correctness of the simulation programs. The most effective way of conducting the latter is to use good, thoroughly tested PRNGs and to control statistical errors of simulation results by analysing them sequentially, i.e. to control the magnitude of the statistical errors of the results by stopping the simulation when the errors of the results reach a satisfactorily low level.

Neglecting the proper statistical analysis of simulation output data cannot be justified by the fact that some stochastic simulation studies, in particular those aimed at evaluating simulated systems in their steady-state, might require sophisticated statistical techniques. On the other hand, it is true that in some cases of practical interest, appropriate statistical techniques have not yet been developed. However, if this is the case, then one should not pretend that he/she is executing a precise quantitative study of the performance of a telecommunication network.

\section{Final Comments}

In this paper we have considered two important necessary conditions of credible simulation studies: use of appropriate pseudo-random generators of independent uniformly distributed numbers, and appropriate analysis of simulation output data. Our survey of recent research publications in the area of telecommunication networks suggests that the majority of recently published results of simulation studies do not satisfy the basic criteria of credibility. The situation could be easily corrected if scientists and engineers who apply stochastic simulation as the tool for studying performance of networks accept fuller responsibility for credibility of their results. An adoption of the basic guidelines indicated in the previous section would be the first step in this direction.

Of course, simulations of telecommunication networks are often computationally intensive and can require long runs in order to obtain results at a desired level of precision. Excessive run-time hinders development and validation of simulation models. Research on speeding up the execution of simulation of telecommunication networks is one of the challenging problems which has attracted considerable scientific interest and effort.

One direction of research in this area has focused on developing methods for the concurrent execution of loosely-coupled parts of large simulation models on multi-processor computers, or multiple computers of a network. Sophisticated techniques have been proposed to solve this and related problems, surveyed, for example, in [1]. In addition to efficiently managing the execution of large partitioned simulation models, this approach can also offer a reasonable speedup of simulation, provided that a given simulation model is sufficiently decomposable. Unfortunately, this feature is not frequently observed in practice, thus the efficiency of this kind of distributed simulation is strongly model-dependent.

In the context of stochastic simulation, there are still at least two additional approaches possible for speeding up such simulations. One of them is based on Variance Reduction Techniques (VRTs); see [12] for a survey of these. No universally applicable VRT can be found. However, such VRTs as Importance Sampling or Splitting (or Restart) can give spectacular speedup in the case of rare event simulation; see for example [29].

Stochastic simulation can also be speeded up by the concurrent generation of multiple, statistically identical streams of output data, using multiple simulation engines running statistically independent replications of simulation processes. This approach to distributed stochastic simulation is known as Multiple Replications In Parallel (MRIP) [23]. A fully automated methodology 
of sequential stochastic simulation in an MRIP scenario has been developed in the Akaroa project at the University of Canterbury, Christchurch, New Zealand. It has resulted in Akaroa-2 [3], a prototype simulation controller that, in its latest version, automatically launches multiple simulation engines and executes terminating or steady-state simulations with full control of statistical errors, in the case of such estimators as mean values, proportions and quantiles. Further increase of the functionality of this type of simulation packages will be possible when some theoretical problems, related to the properties of new (linear combinations of) estimators, are successfully resolved.

\section{Acknowledgements}

The authors would like to thank Dr. Don McNickle for his contributions in research activities that led to this paper, and three anonymous referees for their constructive comments.

\section{References}

[1] Bagrodia. R. L. "Perils and Pitfalls of Parallel Discrete Event Simulation". Proceeding of the 1996 Winter Simulation Conf. WSC’96 (Coronado, California, Dec. 1996). IEEE Press, 1996, 136-143.

[2] Entacher, K. "A Collection of Selected Pseudo-Random Number Generators with Linear Structures". Tech. Report Series Nr. ACPC/TR 97-1, Austrian Center for Parallel Computation, September 1997.

[3] Ewing, G., K. Pawlikowski and D. McNickle. "Akaroa 2: Exploiting Network Computing by Distributed Stochastic Simulation”. Proceedings of the European Simulation Multiconference, ESM'99 (Warsaw, Poland, June 1999). ISCS, 1999, 175-181.

[4] Fitzek, F. H. P., E. Mota, E. Ewers, K. Pawlikowski and A. Wolisz. "An Efficient Approach for Speeding Up Simulation of Wireless Networks". Proc. of the Western Multiconf. on Computer Simulation, WMSC'2000 (San Diego, USA). In press.

[5] Gaither, B. "Empty Empiricism”. ACM Performance Evaluation Review. 18, 1990, no.2, 2-3.

[6] Goldsman, D.; L. W. Schruben and J. J. Swain. "Tests for Transient Means in Simulated Time Series". Naval Research Logistics, 41, 1994, 171-187.

[7] Hellekalek, P. “Don't Trust Parallel Monte Carlo!". Proceedings of the 12th Workshop on Parallel and Distributed Simulation, PADS'98 (Banff, Canada, May 1998). IEEE Computer Society, 1998, 82-89.

[8] Jain, R. The Art of Computer Systems Performance Analysis. Wiley, New York, 1991.

[9] Kiviat, P. J. "Simulation, Technology and the Decision Process". ACM Trans. on Modeling and Computer Simulation, 1, no.2, 1991, 89-98.

[10] Kleijnen, J. P. C. “The Role of Statistical Methodology in Simulation”. In Methodology in Systems Modelling and Simulation, B.P.Zeigler et al., eds. North-Holland, Amsterdam, 1979.

[11] Knuth, D. E. Art of Programming, Volume 2: Seminumerical Algorithms (3rd edition). AddisonWesley, Reading, 1998. 
[12] Law, A. M. and W. D. Kelton. Simulation Modelling and Analysis. Third Edition McGraw-Hill, New York, 2000.

[13] Law, A. M. and M. G. McComas. "Secrets of Successful Simulation Studies". Proceedings of the 1991 Winter Simulation Conf., WSC'91, IEEE Press, 21-27.

[14] L'Ecuyer, P. "Good Parameters and Implementations for Combined Multiple Recursive Random Number Generators". Operations Research, 47, 1999, 159-164.

[15] L'Ecuyer, P. "On the Interaction of Birthday Spacing Tests with Certain Families of Tests of Random Number Generators". Mathematics and Computers in Simulation, to appear.

[16] Lee, Quak Yoong. 1991. "Performance Evaluation of Enhancements to Distributed Queue Dual Bus Protocol". M.E. thesis, Dept. of Electrical and Electronic Eng., University of Canterbury, Christchurch, New Zealand, 1991.

[17] Lee, L. The Day the Phones Stopped: How People Get Hurt When Computers Go Wrong. Donald I. Fine Inc., 1992.

[18] Leeb, H. and S. Wegenkittl. "Inversive and Linear Congruential Pseudo-Random Generators in Empirical Tests". ACM Trans. on Modeling and Computer Simulation, 7, no.2, 1997, 272-286.

[19] Lewis, P.H. "With 2 Chips, the GigaHertz Decade Begins". The New York Times, March 9, 2000.

[20] Matsumoto, M. and T. Nishimura. "Mersenne Twister: a 623-Dimensionally Equidistributed Uniform Pseudo-Random Number Generator". ACM Trans. on Modeling and Computer Simulation, 8, no.1, 1998, 3-30.

[21] Newton-Smith, W. H. The Rationality of Science. Routledge \& Kegan Paul, Boston, 1981.

[22] Pawlikowski, K. "Steady-State Simulation of Queueing Processes: A Survey of Problems and Solutions". ACM Computing Surveys, 2, 1990, 23-170.

[23] Pawlikowski, K.; V. Yau and D. McNickle. "Distributed Stochastic Discrete-Event Simulation in Parallel Times Streams”. Proceedings of the Winter Simulation Conf., WSC'94 (Orlando, Fl., Dec.1994). IEEE Press, 1994, 723-730.

[24] Pawlikowski, K.; D. McNickle and G. Ewing. "Coverage of Confidence Intervals in Sequential Steady-State Simulation". Simulation Practice and Theory, 6, no.2, 1998, 255-267.

[25] Popper, K. R. Realism and the Aim of Science. Hutchison, London, 1983.

[26] Raatikainen, K. E. E. "Sequential Procedure for Simultaneous Estimation of Several Percentiles". Trans. Society for Computer Simulation, 1, 1990, 21-44.

[27] Rezvan, M. "Improving VBR Voice Performance in Integrated Services Broadband Wireless Networks". M.Sc. thesis, Dept. of Computer Science, University of Canterbury, Christchurch, New Zealand, 1998.

[28] Stacey, C.; K. Pawlikowski and D. McNickle. "Detection and Significance of the Initial transient Period in Quantitative Steady-State Simulation". Proceedings of the 8th Australian Teletraffic Research Seminar, ATRS'93 (Melbourne, Australia, Dec. 1993). RMIT, Telstra, 1993, 193-202. 
[29] Townsend, J. K.; Z. Haraszti, J. A. Freebersyser and M. Devetsikiotis. "Simulation of Rare Events in Communication Networks". IEEE Communications Magazine, 36, 8, 1998, 36-41.

\section{BIOGRAPHY}

Krzysztof Pawlikowski is an Associate Professor (Reader) in Computer Science at the University of Canterbury, Christchurch, New Zealand. He received his PhD in Computer Engineering from the Technical University of Gdansk, Poland. The author of over 100 research papers and four books; has given invited lectures at about 100 universities and research institutes in Asia, Australia, Europe and North America. His research interests include stochastic simulation, distributed processing, performance modelling of telecommunication networks (including Internet, ATM, optical and wireless technology) and teletraffic modelling. Senior member of IEEE.

H.-D. Joshua Jeong is currently a PhD student in Computer Science from the University of Canterbury, Christchurch, New Zealand and a student member of IEEE. He received a B.Sc. in Computer Science and Statistics; and an M.Sc. in Computer Science from the Chungnam National University, Taejon, South Korea. He was a research assistant and senior programmer at the same institute from 1986 to 1993. His research interests include teletraffic modelling in telecommunication networks, stochastic simulation and multimedia.

J.-S. Ruth Lee is currently a PhD student in Computer Science from the University of Canterbury, Christchurch, New Zealand and a student member of IEEE. She received a B.Sc. in Computer Science and Statistics; and an M.Sc. in Computer Science from the Chungnam National University, Taejon, South Korea. She was a researcher at the Electronics and Telecommunications Research Institute (ETRI), Taeduck Science Town, South Korea. Her research interests include sequential quantitative stochastic simulation, distributed simulation and stochastic simulation of telecommunication networks. 